\title{
En niños con antecedentes familiares de enfermedad celiaca, la introducción tardía de alimentos con gluten no disminuye el riesgo de desamollarla
}

The late introduction of food with gluten in children with celiac relatives does not decrease the risk of developing the disease

Lionetti E y col. N Engl J Med 2014;371:1295303.

\section{Objetivo}

Establecer la asociación entre el momento de introducción de alimentos con gluten, y el desarrollo de autoinmunidad (AI) y/o enfermedad celíaca (EC) en niños con antecedentes familiares de celiaquía en familiares de primer grado.

\section{Diseño, lugar y participantes}

Ensayo clínico, controlado y aleatorizado, realizado en 20 centros de Italia. Entre 2003 y 2008 fueron reclutados 832 recién nacidos con al menos un familiar de primer grado con EC. Estos fueron asignados en forma aleatoria a alguna de las siguientes ramas: a) introducción de alimentos con gluten a partir de los seis meses; b) introducción de alimentos con gluten a partir de los 12 meses.

\section{Medición de resultados principales}

De acuerdo a su HLA (del inglés human leucocyte antigen), los participantes fueron clasificados en dos grupos según su ries- go de desarrollo de $\mathrm{Al}$ y de $\mathrm{EC}$ a los cinco años de vida: riesgo estándar (HLA-RE) o riesgo alto (HLA-RA). Fueron excluidos del análisis final los 154 niños $(21,8 \%)$ cuyo HLA les confería bajísimo riesgo de desarrollo de EC.

Se definió como resultados principales al desarrollo de anticuerpos (antigliadina, antiendomisio, antitransglutaminasa tipo 2) y/o a la positividad para hallazgos compatibles con enfermedad celíaca de una biopsia intestinal a los dos y cinco años de edad.

\section{Resultados principales}

Un total de 707 niños finalizaron el protocolo (379 en el grupo intervención temprana y 328 en el grupo intervención tardía) con una mediana de seguimiento de 7,9 años. El grupo final estudiado incluyó a 553 niños positivos para HLA-DQ2, HLADQ8 o ambos. Ver resultados en la tabla 1.

Tabla 1. incidencia acumulada a los dos y cinco años de vida de documentación de hallazgos compatibles con enfermedad celíaca en la biopsia intestinal y de evidencia serológica de autoinmunidad en niños con familiares con enfermedad celíaca.

\begin{tabular}{|c|c|c|c|c|}
\hline \multirow[b]{2}{*}{ Tiempo de seguimiento } & \multicolumn{2}{|c|}{$\begin{array}{l}\text { Hallazgos de acuerdo al momento de la introducción } \\
\text { de alimentos con gluten }\end{array}$} & \multirow{2}{*}{$\begin{array}{l}\text { Hazard ratio } \\
\text { (IC95\%) }\end{array}$} & \multirow[t]{2}{*}{$\mathbf{p}$} \\
\hline & $\begin{array}{c}\text { Temprana } \\
\text { (a partir de los } 6 \text { meses) }\end{array}$ & $\begin{array}{c}\text { Tardía } \\
\text { (a partir de los } 12 \text { meses) }\end{array}$ & & \\
\hline \multicolumn{5}{|l|}{ A los dos años } \\
\hline Evidencia serológica de autoinmunidad & $16 \%$ & $7 \%$ & No informado & 0,002 \\
\hline Diagnóstico histológico de celiaquía & $12 \%$ & $5 \%$ & No informado & 0,01 \\
\hline \multicolumn{5}{|l|}{ A los 5 años } \\
\hline Evidencia serológica de autoinmunidad & $21 \%$ & $20 \%$ & $0,9(0,6$ a 1,3$)$ & 0,59 \\
\hline Diagnóstico histológico de celiaquía & $16 \%$ & $16 \%$ & $0,9(0,6$ a 1,4$)$ & 0,78 \\
\hline
\end{tabular}

Ninguna otra variable, incluyendo lactancia materna, mostró estar asociada con el desarrollo de EC.

Como era de esperar, independientemente del momento de la introducción de los alimentos con glúten se constataron diferencias estadísticamente significativas (HR 0,5; IC95\% 0,3 a $0,7)$ a los cinco años en la incidencia acumulada de autoinmunidad entre los niños del subgrupo de alto riesgo por HLA $(38 \%)$ y los del de riesgo estándar (19\%); y una tendencia estadísticamente no significativa de una mayor incidencia de positividad en las biopsias intestinales en el subgrupo de alto riesgo (26 vs $16 \%$ ).

\section{Conclusiones}

La introducción tardía de alimentos con gluten no modificó el riesgo de desarrollar $E C$ en niños con riesgo estándar y alto de EC (por HLA) con familiares de primer grado con EC. Sin embargo, esta conducta sí se asoció a un retraso en la aparición las manifestaciones subclínicas de autoinmunidad (serológicas e histológicas), lo que podría tener algún efecto beneficioso.

Fuente de financiamiento/conflicto de interés de los autores: Fundación Celiachia de la Sociedad Italiana de Enfermedad Celíaca. No refieren conflicto de interés.

¡Vos también podes formar parte de esta transformación!

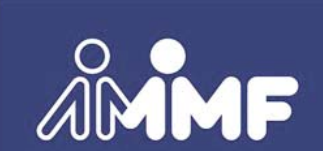

Asociación Metropolitana de Medicina Familiar.

Si queres asociarte o que te brindemos mayor información: secretaria.ammf@gmail.com

Seguinos en Facebook: www.facebook.com/AsociacionMetropolitanadeMedicinaFamiliar 


\section{Comentario}

Si bien no parece haber sesgado los resultados de la investigación, vale destacar que hubo una pérdida en el seguimiento del $15 \%$ de los participantes, la mayoría asignados a la rama de introducción tardía de alimentos con gluten, probablemente por la dificultad de sostener una dieta libre de gluten en ausencia de manifestaciones clínicas de EC.

El estudio finaliza sugiriendo que el conocer el HLADQ del individuo (existe una importante correlación entre la portación de haplotipos de HLA-DQ2 y HLA-DQ8 y el desarrollo en EC) sería de gran utilidad para determinar conductas sobre el seguimiento en aquellos con familiares de primer grado con EC. Sin embargo, en Argentina, la pesquisa de esta enfermedad no es una práctica habitual, por lo que la determinación del HLA-DQ debiera considerarse sólo en casos seleccionados.

\section{Conclusiones de la comentadora}

Previamente a darse a conocer los resultados de este estudio, se consideraba la existencia de una "ventana terapéutica" entre los cuatro y los siete meses de vida, en la cual la introducción de alimentos con gluten modificaría el riesgo de desarrollar EC $^{1,2}$. También se creía que la lactancia materna durante la incorporación de estos alimentos gatillo tendría un efecto protector $^{3,4}$. Los resultados de este estudio debilitan estas hipótesis. Sin embargo la introducción tardía de alimentos con gluten se asoció a un retraso en la aparición de estas manifestaciones subclínicas de EC, concepto que puede no ser menor e invita a la realización de nuevos ensayos diseñados para medir el impacto de este suceso.

Juliana Camoirano [ Servicio de Medicina Familiar y Comunitaria del Hospital Italiano de Buenos Aires, juliana.camoirano@hospitalitaliano.org.ar ]

Camoirano J. En niños con antecedentes familiares de enfermedad celíaca, la introducción tardía de alimentos con gluten no disminuye el riesgo de desarrollarla. Evid Act Pract Ambul. 2016;19(1):19-20. Ene-Mar. Comentado de: Lionetti E, y col. Introduction of gluten, HLA status, and the risk of celiac disease in children. NEJM. 2014;371(14):1295-303. PMID: 25271602.

\section{Referencias}

1. Norris $\mathrm{J}$ y col. Timing of initial cereal exposure in infancy and risk of islet autoimmunity. JAMA. 2003; 290:1713-20.

2. Agostoni C y col. Complementary feeding: a commentary by the ESPGHAN Committee on Nutrition. J Pediatr Gastroenterol Nutr 2008; 46:99-110.

3. Ivarsson A y col. Epidemic of coeliac disease in Swedish children. Acta Paediatr 2000; 89:165-71.

4. Ivarsson A y col. Prevalence of childhood celiac disease and changes in infant feeding. 2013;131: e68794.

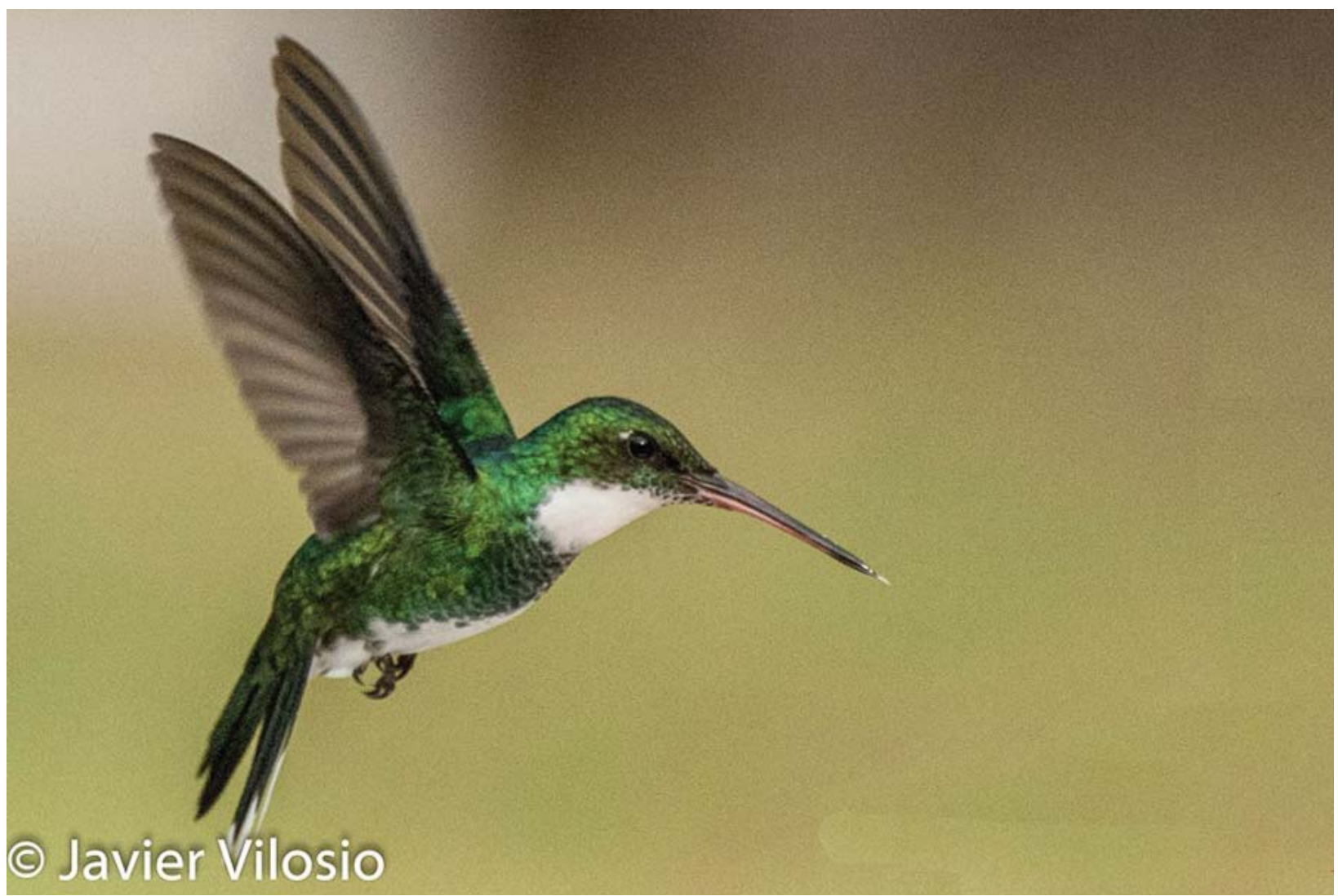

Fotografía: Javier Vilosio 